,Nähe bedeutet auch Zugänglichkeit, Aufgeschlossenheit, Empfänglichkeit für andere. Sie setzt die Begegnung auf Augenhöhe, eine Leichtigkeit der Konsultation, eine gewisse Unmittelbarkeit in der Beziehung, das Fehlen von Förmlichkeit voraus. In der Politik gilt eine Instanz als bürgernah, wenn sie sich nicht auf die Befugnisse versteift, von ihrem Podest steigt, Widerspruch und Kritik akzeptiert, zur Äußerung von Meinungen ermuntert; Wenn sie kurz gesagt, weiß, dass sie nicht auf die institutionellen Abläufe pochen kann und einen flexibleren und direkteren Umgang mit den Bürgern einführen muss." ${ }^{103}$

Der französische Demokratie-Analytiker Pierre Rosanvallon verweist damit auf Ansätze, die schon aufgegriffen wurden, ${ }^{104}$ die aber heute vor allem durch die Digitalisierungsbrille gesehen werden und damit mehr Entfremdung als Nähe und Zusammenhalt bewirken dürften. ${ }^{105}$ Auch Sicherheit kann nicht allein objektiv bewertet werden. ${ }^{106}$ Das Sicherheitsgefühl basiert auf Vertrauen, das sich insbesondere durch Nähe vermittelt. ${ }^{107}$

Schließlich ist auf die ausstehende dritte Verordnung zu Dokumentationspflichten nach $\$ 38$ StandAG zu verweisen, die in inhaltlichem Zusammenhang zu dieser Artikelverordnung steht. Auch dafür ist eine Öffentlichkeitsbeteiligung zu erwägen.

Open Access. Dieser Artikel wird unter der Creative Commons Namensnennung 4.0 International Lizenz veröffentlicht, welche die Nutzung, Vervielfältigung, Bearbeitung, Verbreitung und Wieder- gabe in jeglichem Medium und Format erlaubt, sofern Sie den/die ursprünglichen Autor(en) und die Quelle ordnungsgemäß nennen, einen Link zur Creative Commons Lizenz beifügen und angeben, ob Änderungen vorgenommen wurden.

Die in diesem Artikel enthaltenen Bilder und sonstiges Drittmaterial unterliegen ebenfalls der genannten Creative Commons Lizenz, sofern sich aus der Abbildungslegende nichts anderes ergibt. Sofern das betreffende Material nicht unter der genannten Creative Commons Lizenz steht und die betreffende Handlung nicht nach gesetzlichen Vorschriften erlaubt ist, ist für die oben aufgeführten Weiterverwendungen des Materials die Einwilligung des jeweiligen Rechteinhabers einzuholen.

Weitere Details zur Lizenz entnehmen Sie bitte der Lizenzinformation auf http://creativecommons.org/licenses/by/4.0/deed.de.

Open Access funding enabled and organized by Projekt DEAL.

103) Rosanvallon, Demokratische Legitimität, 2010, S. 250

104) Hoffmann-Riem (Hrsg.), Bürgernahe Verwaltung? 1979

105) Jäger/Lohse/Wissuwa, FAZ v. 21.4.2020, S. 3.

106) Marti, Risikoansichten, ENTRIA-Arbeitsbericht-05, 2016 , S. $36 \mathrm{f}$

107) Frevert, Vertrauensfragen, 2013, S. 211; Smeddinck, in: ders. (Hrsg.), Emotionen bei der Realisierung eines Endlagers, 2018 , S. 11, 18 f.m.w. N.; Schaaf, ,Wir müssen zu einem Wir werden", Interview mit Jutta Allmendinger und Jan Wetzel, FAS v. 8.3.2020, S. 12.

\title{
https://doi.org/10.1007/s10357-021-3837-z \\ Ein Landwirtschaftsgesetz für Deutschland im Zeichen des Umweltschutzes und der Biodiversität - Zentrale Gesetzesinhalte und Finanzierung
}

\section{Detlef Czybulka/Peter Fischer-Hüftle/Ulrich Hampicke/Wolfgang Köck/José Martinez}

() Der/die Autor(en) 2021. Dieser Artikel ist eine Open-Access-Publikation.

Nachdem bereits in einer Abhandlung (NuR 2021, Heft 4) ${ }^{1}$ Notwendigkeit, Funktion und Leitbild eines neuen Landwirtschaftsgesetzes dargestellt wurden, behandelt der vorliegende Aufsatz die er-

\section{Prof. Dr. Detlef Czybulka, Universität Rostock,}

Vorsitzender des Deutschen Naturschutzrechtstages e. V.,

Rostock, Deutschland

Peter Fischer-Hüftle, Vors. Richter am VG a.D.,

Rechtsanwalt, Regensburg, Deutschland

Prof. Dr. Ulrich Hampicke,

ehemals Lehrstuhl für Landschaftsökonomie,

Universität Greifswald, Greifswald, Deutschland

Prof. Dr. Wolfgang Köck,

Mitglied des Sachverständigenrates für Umweltfragen (SRU),

Leiter des Departments für Umwelt- und Planungsrecht am Helmholtz-Zentrum für Umweltforschung - UFZ,

Leipzig, Deutschland

Prof. Dr. José Martinez, Geschäftsführender Direktor des Instituts für Landwirtschaftsrecht der Universität Göttingen,

Mitglied des Wissenschaftlichen Beirats für Agrarpolitik,

Ernährung und gesundheitlichen Verbraucherschutz beim BMEL

sowie Zweiter Vorsitzender der Deutschen Gesellschaft

für Agrarrecht, Göttingen, Deutschland forderlichen materiellen Regelungen, insbesondere zum Schutz der Biodiversität, sowie die Finanzierung.

1. Integration der Anforderungen von Naturschutz (Biodiversität) und Landschaftspflege

\subsection{Problemstellung}

Die landwirtschaftliche Bodennutzung beansprucht unmittelbar die Hälfte unseres Staatsgebiets. Die Auswirkungen von Dünger, Pestiziden und Ammoniak erfassen noch weitere Flächen wie Wälder, Schutzgebiete, ungenutzte Biotope, Gewässer usw. und beeinflussen erheblich den Naturhaushalt und die Biodiversität. Hinzu kommt eine - insbesondere durch Flurbereinigung herbeigeführte und in weiten Teilen Deutschlands vorherrschende - ,ausgeräumte" Landschaft. Sie ist arm an natürlichen Elementen und ungenutzten Flächen. Vor diesem Hintergrund verlangen nicht nur der Schutz von Boden, Wasser, Luft und

1) Dieser trägt den vollständigen Titel „Ein Landwirtschaftsgesetz für Deutschland im Zeichen von Umweltschutz und Biodiversität - Notwendigkeit, Funktion und Leitbild" und ist gemeint, wenn nachfolgend auf „NuR 2021, Heft 4“ verwiesen wird. 
Klima², sondern auch die flächendeckend geltenden Ziele des Naturschutzes ( $\$ 1$ BNatSchG) nach einer wirksamen Berücksichtigung im Rahmen der landwirtschaftlichen Bodennutzung. Daran fehlt es bisher trotz des Auftrags in Art. 20a GG ${ }^{3}$ weitgehend.

Die LANA forderte bereits vor 30 Jahren die langfristige Sicherung aller ökologisch und natur- bzw. kulturhistorisch schutzwürdigen Biotope oder Landschaftsbestandteile sowie die Schaffung eines großräumigen und engmaschigen Biotopverbundes und vielfältiger Landschaftsstrukturen. ${ }^{4}$ In dieselbe Richtung wies 1985 der Sachverständigenrat für Umweltfragen. ${ }^{5}$ Art. 10 FFH-RL fordert seit 1992 die Mitgliedstaaten auf, ,,im Rahmen ihrer Landnutzungsund Entwicklungspolitik, insbesondere zur Verbesserung der ökologischen Kohärenz von Natura 2000“ die Pflege entsprechender Landschaftselemente zu fördern. ${ }^{6}$ Die biodiversitätserhaltende Landschaftspflege mag früher keine landwirtschaftliche Tätigkeit im engeren Sinne gewesen sein, sie gehört aber in der heutigen Situation zum Aufgabenbereich der landwirtschaftlichen Betriebe. ${ }^{7}$

Dabei ist $\mathrm{zu}$ berücksichtigen: Landwirtschaft ist in vielen Ländern die wesentliche Form der Flächennutzung. Aus diesem Grund muss zwischen den unterschiedlichen Nutzungsformen und dem Natur- und Landschaftsschutz im Hinblick auf eine nachhaltige Entwicklung eine enge Verbindung etabliert werden. Diese enge Verknüpfung von strukturellen und funktionalen Aspekten des Naturhaushaltes geht über früher übliche Naturschutzansätze hinaus. ${ }^{8}$ Die Erhaltung und Wiederherstellung der Biodiversität ist ein wichtiges Ziel des Natur- und Landschaftsschutzes. Im Bereich des Naturschutzes existieren daher schon seit vielen Jahrzehnten differenzierte Ansätze zur Erfassung der Biodiversität von natürlichen, naturnahen oder durch den Menschen beeinflussten Ökosystemen. ${ }^{9}$ Im internationalen Vergleich werden am häufigsten verschiedene Vogelarten regelmäßig überwacht. Aber auch unterschiedlichste Gruppen von Wirbellosen oder Insekten dienen als Naturindikatoren zur Messung der Umweltbedingungen. ${ }^{10}$

Rechtlich nicht ausreichend beachtet blieben bislang auch zentrale Aspekte wie Strukturvielfalt, Bedeutung von Korridoren und Pufferzonen sowie die Frage der Repräsentanz bestimmter Arten oder Lebensgemeinschaften innerhalb eines Naturraumes. Die ökologische Bilanzierung landwirtschaftlich genutzter Flächen unter Einschluss der Biodiversität erfordert daher die interdisziplinäre Zusammenarbeit zwischen Agrarexperten, Biologen und Landschaftsökologen. Zweifelsfrei muss die Bilanzierung umweltverträglicher landwirtschaftlicher Nutzung immer den Aspekt der biologischen Vielfalt einschlieBen. Dabei muss die Bilanzierung der biologischen Vielfalt sich grundsätzlich am Erfordernis von Mindestwertgrößen für standorttypische, halb-natürliche Habitate mit nachweislich hohem Biodiversitätswert orientieren. Zugleich sind für die Ermittlung von Mindestwertgrößen für derartige Habitate qualitative und quantitative Mindestansprüche zu definieren, welche den ökologischen Wert für Fauna und Flora im landschaftsökologischen Gesamtraum berücksichtigen.

Eine besondere Herausforderung ist dabei aus landwirtschaftlicher Sicht, ob die teilweise extrem langfristigen Prozesse (wie z. B. die Veränderung des Humusgehaltes des Bodens als Voraussetzung für die pflanzliche Biodiversität) ausreichend genau durch die jetzt diskutierten Indikatoren abgebildet werden können.

\subsection{Maßnahmen zur Förderung der Biodiversität}

Artenvielfalt und Biodiversität benötigen zu ihrer Entfaltung Raum, der ihnen infolge der immer intensiveren Flächennutzung nicht ausreichend zur Verfügung steht. ${ }^{11} \mathrm{Da}-$ raus ergeben sich folgende Prioritäten:

1. Verbliebene Reste der früheren Naturlandschaft wie z.B. Moore sind zu erhalten.

2. Halbkulturlandschaften (Heiden, Magerrasen mit zahlreichen gefährdeten Pflanzen- und Tierarten) sind durch traditionelle Nutzung, vor allem (Schaf-)Beweidung, dauerhaft zu pflegen.

3. Ein Teil der landwirtschaftlichen Nutzflächen, in erster Linie Grünland, ist mit verringerter Intensität zu bewirtschaften. Unumgänglich ist die Wiederherstellung blütenreichen Grünlandes in hoch produktiven Milcherzeugungsgebieten. Wie hervorragende Beispiele in der Eifel zeigen, ${ }^{12}$ ist solches Grünland durchaus in leistungsstarke Betriebe zu integrieren, wenn es der Fütterung nicht der laktierenden Kühe, sondern der Nachzucht dient. Ohne die Wiederherstellung herkömmlicher „Blumenwiesen“ in hinreichendem Umfang (auch fragmentarisch als Wegraine usw.) wird es keine Erholung der Insektenpopulationen geben.

4. Entsprechend ist mit ausgewählten Ackerflächen zu verfahren, die - vorzugsweise auf Kalk- oder Sandboden - ohne Pestizide und mit geringerer Ertragserwartung bewirtschaftet werden.

5. Die Anwendung von Agrarchemikalien mit starker Schadwirkung auf schützenswerte Tierarten wie z. B. Insekten (Neonicotinoide) ist zu beenden, die Risikobewertung für die Zulassung solcher Pflanzenschutzmittel an die neuen Erkenntnisse anzupassen. ${ }^{13}$

6. In der Feldflur sind ausreichende ökologische Vorrangflächen zu schaffen und zu vernetzen wie Brache- und Blühflächen, Pufferstreifen sowie Landschaftselemente wie Kleingewässer, Hecken und Feldgehölze und Wegsäume. Damit lässt sich auch die Schlaggröße auf ein die Biodiversität begünstigendes Maß reduzieren. Spürbare Effekte erfordern einen Anteil von mindestens $10 \%$. Flächenstarke Betriebe müssen auf geringfügigen Flächen wie Ackerrändern und ungünstig geformten Grundstücken Lebensraum für Begleitarten bieten, auf übermäßige „Feldrandhygi-

2) Siehe NuR 2021, Heft 4, zu 5.2.3.

3) Siehe NuR 2021, Heft 4, zu 1.

4) Länderarbeitsgemeinschaft Naturschutz, Landschaftspflege und Erholung, Lübecker Grundsätze des Naturschutzes, 1991; Vgl. die detaillierte Darstellung bei Knickel/Janßen/Schramek/Käppel, in: Bundesamt für Naturschutz (Hrsg.), Angewandte Landschaftsökologie, Heft 41, 2001, S. 45 f. mit Änderungen und Ergänzungen.

5) SRU, Sondergutachten Umweltprobleme der Landwirtschaft, 1985, BT-Drs. 10/3613.

6) Vgl. Czybulka, in: Symank (Bearb.), Vorrangflächen, Schutzgebietssysteme und naturschutzfachliche Bewertung großer Räume in Deutschland, Schriftenreihe für Landschaftspflege und Naturschutz Heft 63, Bundesamt für Naturschutz 2000, S. 169-201.

7) Siehe unten $\mathrm{zu} 4.1$

8) Vgl. Wascher, in: Diepenbrock, Umweltverträgliche Pflanzenproduktion: Indikatoren, Bilanzierungsansätze und ihre Einbindung in Ökobilanzen. Initiativen zum Umweltschutz Bd. 5, 1997.

9) Bereits 1997 wählte der 3. Warnemünder Naturschutzrechtstag das Thema „Ist die biologische Vielfalt zu retten?“ Dazu im Tagungsband, insbes. Müller-Motzfeld, in: Czybulka (Hrsg), Tagungsband 2002, S. $141 \mathrm{ff}$.

10) Dröschmeister/Gruttke, Die Bedeutung ökologischer Langzeitforschung für Naturschutz. Schriftenreihe für Landschaftspflege und Naturschutz, Heft 58, 1998.

11) Vgl. Bundesamt für Naturschutz, Wie die Europäische Agrarpolitik $2020+$ die biologische Vielfalt stärken kann, BfN Pressemitteilung vom 12.8.2020.

12) Schumacher, Bilanz - 20 Jahre Vertragsnaturschutz. NaturschutzMitteilungen 1/07, 2007, S. 21-28; ders., in: Czybulka/Köck (Hrsg.), Landwirtschaft und Naturschutzrecht, 2019, S. 47-64.

13) Vgl. Schäffer et. al., Der stumme Frühling - zur Notwendigkeit eines umweltverträglichen Pflanzenschutzes, Leopoldina Diskussion Nr. 16/2018, S. 35 ff. Für das Zulassungssystem wäre allerdings in erster Linie der europäische Gesetzgeber zuständig; dazu näher Köck/Kern, in: Perspektiven des Stoffrechts, UTR Bd. 114, S. 21, 31-41. 
ene" verzichten und (in der Regel übertriebene) Risiken der Einwanderung von unerwünschten Arten tolerieren.

7. Mehrjährige und lagetreue Brachen sind von herausragender Bedeutung für den Erhalt der Biodiversität in Ackerbaulandschaften. Hinzu kommen Altgrasstreifen und überjährige Streifen sowie Uferrand- und Pufferstreifen, etwa als Rückzugsräume für Insekten, die von dort aus gemähte Flächen wieder besiedeln können. ${ }^{14}$

8. Biotope und Landschaftselemente sind gegen negative Einflüsse aus der Bodenbewirtschaftung zu schützen.

9. Auf schutzbedürftige Arten - von Kiebitz und Kornweihe bis zum Ackerwildkraut Adonisröschen - ist unter fachlicher Betreuung (möglichst aus der Landwirtschaft selbst) bei der Bewirtschaftung Rücksicht zu nehmen. Die von $\$ 44$ Abs. 4 Satz 3 BNatSchG geforderten Bewirtschaftungsvorgaben sind schwer zu handhaben und durch gesetzliche Grundpflichten zu ergänzen wie z.B. Art und Zeitpunkt der Mahd, Zeitpunkt des Walzens von Grünland usw. ${ }^{15}$

10. Oberflächengewässer sind vor Immissionen aller Art (Phosphor- und Stickstoffdünger, Abdrift von Pestiziden) sowie vor schädlichen mechanischen Eingriffen, wie intensiven „Pflegemaßnahmen“ in Gräben, zu schützen.

11. Es ist ein naturnäherer Wasserhaushalt herzustellen. Beim Herauspumpen von Wasser aus der Landschaft zur Herstellung optimaler Bedingungen für den Ackerbau ist nicht zuletzt mit Blick auf den Klimawandel ein Umdenken erforderlich.

12. Erforderlich ist eine starke Reduzierung der Ammoniak $\left(\mathrm{NH}_{3}\right)$-Emissionen in Höhe von noch 680000 Tonnen pro Jahr mit der Folge landesweiter Eutrophierung.

Diese Maßnahmen dienen primär dem Schutz von Flora und Fauna, haben aber weitere wichtige positive Effekte auf die Schönheit und Erholungseignung der Landschaft, Eindämmung der Winderosion, Schutz der Gewässer und andere.

Nicht nur die umweltbezogenen Ziele, ${ }^{16}$ sondern auch die erforderlichen Maßnahmen zur Erhaltung und Förderung der Biodiversität müssen in einem Landwirtschaftsgesetz verankert werden, weil die Probleme sämtlich von der Landwirtschaft verursacht und daraus Pflichten für die landwirtschaftliche Bodennutzung abzuleiten sind. Ihre alleinige Verortung im Naturschutzrecht, verbunden mit dem Vollzug durch Naturschutzbehörden, würde als unwillkommene Einflussnahme von außen nicht die erforderliche Akzeptanz finden. Landwirte und ihre Fachbehörden nehmen Vorschriften eher ernst, wenn sie aus dem eigenen Fachrecht kommen, was langfristig auch zu einer anderen Denkweise führen kann.

Die Ziele und Maßnahmen lassen sich zum Teil dadurch verwirklichen, dass in der Praxis der landwirtschaftlichen Bodennutzung bestimmte Grundanforderungen einzuhalten sind. ${ }^{17}$ Für bestimmte Leistungen kommen finanzielle Anreize in Betracht, z.B. zur pflegenden Bewirtschaftung von Halbkulturlandschaften oder von artenreichem Grünland für Milchviehbetriebe im Mittelgebirge. ${ }^{18}$ Maßnahmen, die auf die dauerhafte oder turnusmäßige Freihaltung von landwirtschaftlichen Flächen zugunsten natürlicher Elemente oder Entwicklungen abzielen oder der Wiederherstellung eines naturnäheren Landschaftswasserhaushaltes dienen, können teilweise auf betrieblicher Ebene verwirklicht werden, etwa die Schaffung oder Pflege von natürlichen Landschaftselementen und Blühflächen. Dagegen erfordern die Herstellung eines Biotopverbunds und wasserwirtschaftliche Maßnahmen in der Regel eine fachliche Planung. Zwecks Förderfähigkeit sind die Begriffe der landwirtschaftlichen Tätigkeit und Fläche anzupassen. ${ }^{19}$

\section{Grundpflichten bei der landwirtschaftlichen Bodennutzung und Tierhaltung}

\subsection{Notwendigkeit klarer Vorgaben}

Die desaströsen Auswirkungen der heute überwiegend praktizierten Landwirtschaft auf Umwelt und Biodiversität verlangen rechtliche Maßnahmen, die von der Wissenschaft immer wieder formuliert ${ }^{20}$, von der Politik aber nicht aufgegriffen wurden. Dagegen sind „Agrarprivilegien“" keinesfalls mehr zu rechtfertigen. ${ }^{21}$

Die bisherigen Grundsätze der ,guten fachlichen Praxis“ sollen Anforderungen an die Landwirtschaft im Allgemeininteresse des Umwelt- und Naturschutzes stellen. Sie sind aber als bloße Grundsätze zu unbestimmt, sodass sie die ihnen zugedachten Wirkungen zu keinem Zeitpunkt erreichen konnten. ${ }^{22}$ Auch ein verstärkter Rückgriff auf die Eingriffsregelung würde nicht zum Ziel führen: Eine Änderung der Grundflächengestalt oder-nutzung (\$14 Abs. 1 BNatSchG) kann bei vielen Bewirtschaftungstätigkeiten (z.B. Pestizidanwendung, Anzahl von Großvieheinheiten) nicht oder nicht eindeutig bejaht werden. Die Feststellung der Verursacherpflichten ( $\$ 15$ BNatSchG) ist auf Folgenbewältigung im Einzelfall gerichtet ${ }^{23}$ und daher als ex-ante-Handlungsdirektive für die Bewirtschaftung landwirtschaftlicher Flächen ungeeignet.

Die Anforderungen an die landwirtschaftliche Bodennutzung zum Schutz von Natur- und Umweltgütern sowie des Tierwohls müssen als konkrete, unmittelbar geltende Pflichten formuliert sein. ${ }^{24}$ Wenn man sich nicht gänzlich von dem Etikett „Gute fachliche Praxis“"verabschieden will, muss dieser Begriff jedenfalls mit konkreten und verbindlichen Inhalten ausgefüllt werden. Deshalb ist es an der Zeit, diese Grundsätze durch unmittelbar geltende Grundpflichten und hierauf bezogene Konkretisierungsermächtigungen zu ersetzen bzw. auszufüllen. Für den Landwirt muss klar erkennbar sein, was er zu tun oder zu unterlassen hat und wo er eine Förderung erfährt.

Wir unterscheiden dabei zwischen allgemeinen Grundpflichten, ${ }^{25}$ die für alle Landwirte gelten, und spezifischen Pflichten für Landwirte, die in besonders belasteten Räumen oder Entwicklungsgebieten wirtschaften. ${ }^{26}$ Die Erfüllung der allgemeinen Grundpflichten ist durch die Behörden sicherzustellen. ${ }^{27}$ Sie muss Grundvoraussetzung für jede nationale oder europäische Förderung sein und kann ggf. Ausgleichszahlungen rechtfertigen. ${ }^{28}$

14) Dazu näher Oppermann et al., Biodiversität in der Gemeinsamen Agrarpolitik (GAP) der EU nach 2020, Stand 18.3.2021, abrufbar unter https://www.bfn.de/themen/landwirtschaft/veroeffentlichungen.html.

15) Vgl. Art. 3 Abs. 4 Nr. 5-7 BayNatSchG in der Fassung des Volksbegehrens 2019.

16) Siehe NuR 2021, Heft 4, zu 5.2.3.

17) Siehe unten zu 2

18) Siehe unten zu 5

19) Siehe unten zu 4

20) So etwa Möckel/Köck/Rutz/Schramek, Rechtliche und andere Instrumente für vermehrten Umweltschutz in der Landwirtschaft, Gutachten im Auftrag des UBA (UFO-Plan FKZ 371188 191), 2013.

21) Dazu Ekardt, in: Kloepfer (Hrsg.), Umweltschutz als Rechtsprivileg, 2014, S. $111 \mathrm{ff}$

22) Siehe BVerwG, Urt. v. 1.9.2016 - 4 C 4.15, ZUR 2017, 176; Möckel, ZUR 2014, 14.; Köck, in: Köck/Czybulka (Hrsg.), Landwirtschaft und Naturschutzrecht, 2019, 189.

23) BVerwG, Beschl. v. 31.1.1997 - 4 NB 27/96, NuR 1997, 543 Rdnr. 19: „Folgenbewältigungsprogramm“.

24) Konkrete qualitative oder quantitative Anforderungen fordert auch Möckel, ZUR 2014, 14 m. w. N.

25) Siehe unten zu 2.2

26) Siehe unten zu 2.3

27) Siehe unten zu 2.4.3

28) Siehe unten zu 5.5 
Die Parallele zu den Pflichten der Betreiber bestimmter Anlagen bedeutet nicht, dass der Beruf des Landwirts künftig ein Gewerbe ist, sondern lediglich, dass bei der Ausübung der landwirtschaftlichen Nutzung feste Regeln zur Wahrung der in Art. 20a GG verankerten Gemeinwohlinteressen einzuhalten sind. Diese Betreiberpflichten sollen gewährleisten, dass Umweltgüter wie Boden, Wasser, Luft, Klima, Naturhaushalt (einschließlich Biodiversität) und Landschaftsbild sowie bei der Tierhaltung das Tierwohl so wenig wie möglich beeinträchtigt werden. In diesem Kontext ist auch eine präventive Kontrolle vorstellbar, etwa indem - ggf. differenziert nach Lage und Art des landwirtschaftlichen Betriebs - eine Genehmigungs- oder Anzeigepflicht bezüglich bestimmter umweltrelevanter Umstände der aktuellen oder geplanten Bewirtschaftung besteht. ${ }^{29}$

Die Einhaltung der Betreiberpflichten kann im Rahmen der naturschutzrechtlichen Eingriffsregelung als (widerlegbare) Vermutung zugunsten des Landwirts formuliert werden (vgl. $\$ 14$ Abs. 2 BNatSchG).

\subsection{Allgemeine Grundpflichten}

\subsubsection{Einhaltung der guten fachlichen Praxis} und nach Maßgabe gesetzlicher Spezifizierung auch des Standes der Technik

Das Landwirtschaftsgesetz verankert die Konzeption durchsetzbarer ökologisch ausgerichteter Betreiberpflichten/Grundpflichten in den Bereichen Immissionsschutz, Naturschutz, Bodenschutz, Gewässerschutz, Pflanzenschutz, Düngung, Gentechnik/Saatgut, Tierschutz. Es regelt diese zum Teil selbst und enthält im Übrigen dynamische Verweisungen auf die bundes- und landesrechtliche Konkretisierung der Betreiberpflichten in den umweltmedienbezogenen Regelungen. Der Bund kann sich dabei auf verschiedene Umweltkompetenztitel sowie die Zuständigkeit für Bodenrecht und Tierschutzrecht stützen. ${ }^{30}$

Zur Aufnahme in das Landwirtschaftsgesetz eignen sich besonders Regelungen über die Art und Weise der landwirtschaftlichen Bodennutzung, soweit sie Auswirkungen auf $\mathrm{Na}$ tur- und Umweltgüter hat, also spezifisch landwirtschaftliche Aspekte des Bodenschutzes, des Naturschutzes (derzeit $\int 5$ Abs. 2 BNatSchG) und des Gewässerschutzes (wie etwa $\$ 38 \mathrm{a}$ WHG), sowie die Anforderungen an die Haltung von Nutztieren. Die Materie des Düngegesetzes samt Verordnungsermächtigung ist in das Landwirtschaftsgesetz zu überführen. Auch liegt es nahe, die Anwendung von Pflanzenschutzmitteln auf landwirtschaftlich genutzten Flächen im Landwirtschaftsgesetz zu regeln. ${ }^{31}$ Dagegen sollten die planerischen und technischen Anforderungen an Tierhaltungsanlagen im Bauplanungs- bzw. Immissionsschutzrecht verbleiben.

Diese Pflichten lassen sich mit Bezug auf die oben ${ }^{32}$ genannten Maßnahmen wie folgt systematisieren:

1. Verbote, wie z.B. keine Ausbringung unzulässiger Agrarchemikalien, kein Grünlandumbruch, keine rücksichtslose „Grabenpflege“ mit Tod aller Amphibien usw.

2. Gebote zu schonenden Bestell-, Pflege- und Erntemaßnahmen, zum Beispiel Wiesenmahd mit mäßiger Geschwindigkeit, die Tieren Fluchtmöglichkeiten belässt.

3. Gebote zur Nutzung technischer Anlagen und Verfahrensweisen zu Reduzierung von AmmoniakEmissionen, wie Abluftfilter für Ställe und schonende Ausbringungsverfahren für Gülle.

4. Gebote zur Bereitstellung von Flächen zugunsten erforderlicher Strukturelemente.

5. Gebote zur Umwidmung von Teilflächen zugunsten traditioneller und weniger ertragreicher Wirtschaftsweisen.

6. Änderungen der Feuchtigkeitsverhältnisse, insbesondere Anhebungen des Grundwasserstandes in Grün- landgebieten, sind zu tolerieren, wenn dies zur Erhaltung der Lebensgrundlage schutzbedürftiger Arten erforderlich ist.

Die zu (1.) und (2.) genannten Pflichten erfordern keinen finanziellen Ausgleich. Für die Umrüstung von Ställen (3.) kommt eine Förderung in Betracht, um sie zu beschleunigen. Nr. 4, 5 und 6 erfordern in der Regel eine besondere Honorierung. ${ }^{33}$

\subsubsection{Pflichten zur Anpassung an den Klimawandel}

Was die Emissionen von Methan und Stickoxid in der Landwirtschaft betrifft, gelten die Regelungen der Klimaschutzgesetze des Bundes und der Länder und der darauf gestützten Verordnungen. Laut Klimaschutzprogramm 2030 soll die Reduktion der THG-Emissionen durch ein Bündel von Maßnahmen, u. a. auch durch eine Neuausrichtung der Förderpolitik für die Nutztierhaltung, erreicht werden. ${ }^{34}$ Im Übrigen soll insbesondere auch durch die Ausweitung des Ökolandbaus und durch Synergien mit den notwendigen Maßnahmen zur Anpassung an den Klimawandel auf die Reduktion von THG hingewirkt werden. ${ }^{35}$

Während die Klimaschutzmaßnahmen im Wesentlichen über die Klimaschutzgesetze und deren ausführende Programme vorbereitet werden, wäre mit Blick auf die $A n$ passung an den Klimawandel das Landwirtschaftsgesetz der richtige Ort, um im notwendigen Umfang Pflichten festzulegen. Da Anpassungsmaßnahmen nicht nur im Eigeninteresse der Landwirte liegen, sondern wegen des bisherigen Mechanismus der Gewährung staatlicher Hilfen für klimawandelbedingte Ernteausfälle bzw. Ernteeinbußen auch im gesellschaftlichen Interesse sind, sollte eine allgemeine Grundpflicht der Anpassung gesetzlich vorgesehen werden. Vorbilder dazu finden sich bereits in Einzelgesetzen. ${ }^{36}$ Um mehr Wasser in der Landschaft zu halten, kommt eine Duldungspflicht bei Wiedervernässungsmaßnahmen in Betracht. Außerdem sind Torf- und Moorböden nur als Grünland zu bewirtschaften.

\subsubsection{Bildung und Fortbildung}

Die landwirtschaftliche Tätigkeit erfordert bislang rechtlich keine landwirtschaftliche Ausbildung. Nur mittelbar wirkt ein rechtlicher Zwang z. B. über die Bestimmungen der Höfeordnung zur Auswahl des Hoferben. Erforderlich ist nur in begrenzten Bereichen (Pflanzenschutz, Tierschutz) ein Sachkundenachweis. Wichtige Bereiche wie Fruchtfolgen, Düngemittel, Gewässerschutz oder Artenkenntnis sind davon nicht erfasst.

Zwar verfügen die meisten Landwirte über eine landwirtschaftliche Ausbildung, die Vorgaben an die Fortbildung gerade im Hinblick auf die umweltrelevanten Gefahren sind jedoch minimal. Eine Verpflichtung zur Fortbildung jenseits der bestehenden Sachkundenachweise, geregelt in einem Landwirtschaftsgesetz, wäre im Hinblick auf die Gefahrgeneigtheit der landwirtschaftlichen Tätig-

29) Siehe unten zu 2.4.1.

30) Siehe NuR 2021, Heft 4, zu 2.

31) Die geltende Verordnung über Anwendungsverbote für Pflanzenschutzmittel (PflSchAnwV) datiert aus dem Jahr 1992 und verbietet in $\$ 4$ die Anwendung bestimmter Stoffe in naturschutzrechtlichen Schutzgebieten und geschützten Biotopen, wobei Natura 2000-Gebiete nicht erwähnt werden.

32) Siehe zu 1.2.

33) Siehe unten zu 5.

34) Vgl. Bundesregierung, Klimaschutzprogramm 2030, S. 115.

35) Bundesregierung, Klimaschutzprogramm 2030, S. 105 f., 107.

36) Siehe etwa für die Störfallsicherheit von Industrieanlagen die allgemeine Betreiberpflicht des $₫ 3$ Abs. 2 Nr. 2 der 12. BImSchV und die Ermittlungspflichten der UVP (Anl. 3 Ziff. 1.6 UVPG); für die Gewässerbewirtschaftung: $\int 6$ Abs. $1 \mathrm{Nr}$. $5 \mathrm{WHG}$; für die Stadtplanung: $\$ 1$ Abs. 5 BauGB; für die Raumordnung: $\$ 2$ Abs. 2 Nr. 6 ROG. 
keit im Umgang mit den natürlichen Lebensgrundlagen zwingend erforderlich.

Landesrecht sieht vor, dass als Bestandteil der pädagogischen Aus- und Fortbildung, in den Lehr- und Bildungsplänen und bei den Lehr- und Lernmitteln die Ziele und Aufgaben des Naturschutzes und der Landschaftspflege zu berücksichtigen und insbesondere die Folgen des Stickstoffeintrages, die Auswirkungen von Schlaggrößen, die Bedeutung der Fruchtfolge-Entscheidungen und die Auswirkungen des Pestizideinsatzes und weiterer produktionsintegrierter Maßnahmen auf den Artenreichtum und das Bodenleben darzustellen sind. ${ }^{37}$ Solche Materien müssen auch Gegenstand der Aus- und Fortbildung der Landwirte sein.

\subsection{Spezifische Pflichten für ausgewiesene Belastungs- und Entwicklungsgebiete}

Für ausgewiesene Belastungsgebiete ${ }^{38}$ und spezifische Entwicklungsgebiete können sich Pflichten ergeben, die über die allgemeinen Grundpflichten hinausgehen. Hierbei kann ein generalisierender Ansatz verfolgt werden, indem das Gesetz spezifische Pflichten für solche Gebiete regelt, denkbar und möglicherweise vorzugswürdig ist aber auch, dass spezifische Ge- und Verbote bzw. Handlungspflichten in der Gebietsverordnung festgelegt werden. Für Entwicklungsgebiete sind Biosphärenreservate (als Kerngebiete) wegen ihrer in $\$ 25$ Abs. $1 \mathrm{Nr} .3$ und 4 BNatSchG verankerten Zielstellung besonders geeignet. Spezifische Verpflichtungen in diesen Gebieten können u. U. aus Gründen der Verhältnismäßigkeit, aber auch aus Billigkeitserwägungen finanzielle Ausgleichszahlungen nach sich ziehen. ${ }^{39}$

\subsection{Durchsetzung der Grundpflichten}

\subsubsection{Eröffnungskontrollen}

Angesichts der erheblichen Umwelt- und Biodiversitätsbeeinträchtigungen, die von der Landwirtschaft ausgehen können, erscheint eine präventive Kontrolle sachgerecht und verhältnismäßig. Sie muss nicht stets in einen Genehmigungsvorbehalt münden, denkbar sind auch (sanktionsbewehrte) Anzeigepflichten. Bewirtschaftungsformen wie die Nutzung gentechnisch veränderten Saatguts unterliegen bereits auf gentechnikrechtlicher Grundlage einem Genehmigungsvorbehalt. Die präventive Kontrolle kann, im Lichte der Grundrechtsauswirkungen abgestuft, am Maßstab der Beeinträchtigungen von Natur und Umwelt erweitert werden. Dazu ist das Integrierte Verwaltungs- und Kontrollsystem der EU (InVeKoS) nutzbar zu machen. ${ }^{40}$ Es stellt ein System zur Identifizierung landwirtschaftlich genutzter Parzellen und zur Bearbeitung von Beihilfeanträgen bereit.

Eine bereits bestehende, jedoch in Deutschland aufgrund einer europarechtswidrigen Auslegung kaum genutzte präventive Kontrollmöglichkeit ist die Verträglichkeitsprüfung in Natura 2000-Gebieten. Sie knüpft an den Begriff des Projekts an. Der EuGH sieht in ständiger Rechtsprechung landwirtschaftliche Bewirtschaftungstätigkeiten, zuletzt auch im Hinblick auf Weidehaltung und Düngung, ${ }^{41}$ als Projekt an und verlangt damit eine Verträglichkeitsprüfung, wenn Beeinträchtigungen der Schutzziele des Natura 2000-Gebiets nicht sicher ausgeschlossen werden können. Hier wäre eine europarechtskonforme Klarstellung im Landwirtschaftsgesetz dahingehend, dass eine Bewirtschaftung den Projektbegriff des FFH-Rechts erfüllen kann, nützlich. ${ }^{42}$ Da die FFH-Verträglichkeitsprüfung wirkungsbezogen ist, kann sie auch landwirtschaftliche Nutzflächen außerhalb der Natura 2000-Gebietskulisse betreffen, soweit die Bewirtschaftung erhebliche Auswirkungen auf die Erhaltungsziele eines benachbarten Natura 2000-Gebiets haben kann.

\subsubsection{Verordnungsermächtigungen}

Wo immer möglich, sollte der Gesetzgeber unmittelbar geltende und vollziehbare Regelungen schaffen, an die sich der Landwirt ohne weitere Zwischenschritte halten muss. Doch eignet sich nicht alles für eine Regelung durch Gesetz, etwa wenn es auf die besonderen Verhältnisse in bestimmten Gebieten ankommt. Verordnungsermächtigungen sollten mit einer Verpflichtung zur Regelung innerhalb bestimmter Frist verbunden sein, um ein konsistentes Normengefüge ohne Lücken zu gewährleisten. Dies gilt auch dann, wenn der Landesgesetzgeber zur weiteren Konkretisierung ermächtigt wird.

\subsection{3 Überwachung und Befugnisse der Behörden}

Das notwendige Gegenstück zu den Betreiberpflichten bildet die Ermächtigung der Behörde zu deren Durchsetzung. Hier bietet sich eine Generalklausel an, etwa nach dem Vorbild von $\$ 3$ Abs. 2 BNatSchG, wonach die zuständigen Behörden die Einhaltung der Vorschriften überwachen und nach pflichtgemäßem Ermessen die im Einzelfall erforderlichen Maßnahmen treffen (sollen), um deren Einhaltung sicherzustellen..$^{43}$

Die Entscheidung, wer als Vollzugs- und Kontrollbehörde bestimmt wird, muss sich nach dem sachlichen Inhalt der $\mathrm{zu}$ vollziehenden Regelungen und den vorhandenen Personalkapazitäten richten. Das hat zur Konsequenz, dass die personell gut ausgestatteten Landwirtschaftsbehörden aus ihrer (bequemen) Berater- und Unterstützungsrolle herauswachsen müssen und die (unterbesetzten) Naturschutzbehörden ${ }^{44}$ nicht überlastet werden dürfen. Die Bedenken dagegen von Naturschutzseite sind bekannt, es besteht aber keine realistische Alternative.

\subsection{Vorgaben für die Nutztierhaltung}

Das Tierschutzgesetz enthält keine Vorgaben für eine Nutztierhaltung, die die Besonderheiten des Nutztier-Tierschutzrechtes definieren. Diese müssen daher gesondert herausgearbeitet werden und aufgrund ihrer Wirkung jenseits des Tierschutzes i.e.S. (z. B. auf das Bau- und Immissionsschutzrecht) durch eine Verortung im Landwirtschaftsgesetz vor die Klammer gezogen werden. Zu diesen Leitlinien gehört die Prämisse der anthroporelational determinierten Nutzbarkeit der Nutztiere sowie das Erfordernis der Gruppenhaltung in begrenzten Arealen. Die Größe und Haltungsmethoden müssen vom Tierwohl und gleichermaßen von ihren ökologischen Wirkungen determiniert werden.

Hier wirken sich die Vorgaben auf die künftige Fortentwicklung des Immissionsschutzrechtes und des Düngerechts aus. Sollte dies nicht ausreichend steuernd wirken, muss die Einführung von regionalen Bestandsobergrenzen als verbindlicher Bestandteil der guten fachlichen Praxis/Betreiberpflichten bereits bundesrechtlich im Landwirtschaftsgesetz ermöglicht werden. ${ }^{45}$ Schließlich ist eine nachhaltige landwirtschaftliche Nutztierhaltung zwin-

37) Art. 1b BayNatSchG in der Fassung des Volksbegehrens 2019.

38) Siehe unten zu 3.2

39) Siehe unten zu 5.

40) Vgl. national Verordnung über die Durchführung von Stützungsregelungen und des Integrierten Verwaltungs- und Kontrollsystems (InVeKoS) v. 24.2.2015, BGBl. I S. 166.

41) EuGH, Urt. v. 7.11.2018 - C-293/17 und C-294/17, NuR 2018, 852.

42) Martinez, DVBl. 2020, 1186, $1190 \mathrm{f}$

43) Diese Generalklausel geht über die Gefahrenabwehr hinaus und umfasst einerseits die Wiederherstellung des früheren Zustands (VGH München, Beschl. v. 9.8.2012 - 14 C 12.308, juris), andererseits Gefahrerforschungsmaßnahmen (OVG Lüneburg, Beschl. v. 26.10.2015 - 4 ME 229/15, NuR 2016, 42).

44) Vgl. das (folgenlose) Sondergutachten des SRU: Für eine Stärkung und Neuorientierung des Naturschutzes, 2002.

45) Schramek et. al., Vorschläge zur Ausgestaltung von Instrumenten für einen effektiven Schutz von Dauergrünland, BfN-Skripten, 2012, S. 56: 1,4 Großvieheinheiten/ha. 
gend an eine konkrete Futtergrundlage zu koppeln. Die seit 2004 in $\$ 201$ BauGB geregelte Entkoppelung wird bereits in Frage gestellt. ${ }^{46}$ Dadurch könnte auch die nach $\$ 35$ Abs. 1 Nr. 1 BauGB bestehende Entkoppelung zwischen dem Betriebszweig Tierhaltung und dem Ackerbau rückgängig gemacht werden.

\subsection{Vereinbarkeit mit dem Eigentumsgrundrecht und der Berufsfreiheit}

Nach dem Selbstverständnis vieler Landwirte mag die Schaffung eines solchen Bündels von Betreiberpflichten als Eingriff in ihre Rechte als Grundeigentümer oder Pächter empfunden werden. Der Aspekt der Situationsgebundenheit von Grundstücken gilt jedoch für alle landwirtschaftlichen Grundstücke insofern, als sie nicht nur Nutzfläche, sondern notwendigerweise und viel stärker als bebaute Flächen auch Teil des Naturhaushalts sind. Die Wirkungen der Düngung und Pestizidanwendung reichen überdies weit über die Nutzflächen hinaus. Das Eigentum und sein Gebrauch wirken sich damit unvermeidlich und erheblich auf Rechtsgüter aus, die im Gemeinwohlinteresse vor Verschlechterung zu schützen sind. Auf gesunde Böden, saubere Luft und ein funktionierendes Agrarökosystem mit hoher Biodiversität sind wir alle angewiesen.

Mit diesem starken Bezug zu den Schutzgütern von Umwelt und Natur verlässt das Eigentum an landwirtschaftlich genutzten Flächen deutlich den Bereich der Privatsphäre. Die zum Grundstücksverkehrsrecht getroffene Feststellung des BVerfG, eine gerechte Rechts- und Gesellschaftsordnung zwinge dazu, die Interessen der Allgemeinheit beim Boden in weit stärkerem Maße zur Geltung zu bringen als bei anderen Vermögensgütern, ${ }^{47}$ gilt ebenso und erst recht in Bezug auf Umwelt und Biodiversität. Der Inhalt des Grundeigentums ist entsprechend zu bestimmen, um darauf bezogene Gemeinwohlziele zu erreichen. Die richterrechtliche „Situationsgebundenheit“ des Eigentums ist fortzuentwickeln zu einer Ökologiepflichtigkeit der Eigentümer in Bezug auf die Nutzung der natürlichen Lebensgrundlagen, deren Schutz Art. 20a GG seit 1994 vorgibt, deren normative Ausformung der Gesetzgeber aber bis heute vernachlässigt hat. ${ }^{48}$

Soweit das Grundrecht aus Art. 12 GG betroffen ist, bilden die Grundpflichten des Landwirts Regelungen der Berufsausübung zur Verfolgung legitimer Zwecke des Gemeinwohls.

\section{Planung und Raumordnung}

\subsection{Allgemeines}

Unter Planung wird ,das vorausschauende Setzen von Zielen und gedankliche Vorwegnehmen der zu ihrer Verwirklichung erforderlichen Verhaltensweisen" verstanden. ${ }^{49}$ Unterschieden wird dabei zwischen Regierungsplanung als (strategische) politische Planung-Agrarpolitischer Bericht der Bundesregierung, ${ }^{50}$ Agrarprogramme oder Aktionsprogramme - und verwaltungsrechtlicher Planung. ${ }^{51}$ Während der Agrarpolitische Bericht als sog. ,,indikativer Plan" ${ }^{\circ 2}$ lediglich über Informationen steuert, bereiten Agrarprogramme z.B. Förderpolitiken sowie Aktionsprogramme Maßnahmen zur Gefahrenabwehr bzw. zur Sanierung vor $^{53}$ und lenken damit (jedoch nicht in ausreichendem Maße) das Verwaltungshandeln. Als verwaltungsrechtliche Planung ist insbesondere die Flurbereinigung bedeutungsvoll, ${ }^{54}$ die allerdings in der alleinigen Zuständigkeit der Länder liegt (Art. 74 Abs. 1 Nr. 17 GG) und deshalb nicht in einem Landwirtschaftsgesetz des Bundes Platz finden kann.

Notwendigkeit und Nutzen einer Planung auf örtlicher oder regionaler Ebene setzen voraus, dass es besondere Umstände gibt, die sich einer generellen, normativen Rege- lung entziehen. Soweit sich bei Handlungen der landwirtschaftlichen Bodennutzung die Probleme im Verhältnis zu den Schutzgütern des Umwelt- und Naturschutzrechts durch konkrete, vollzugsfähig formulierte Rechtsnormen auf Gesetzes- oder Verordnungsebene bewältigen lassen, bedarf es keiner auf lokale Besonderheiten abgestimmten Planung. Diese kann dagegen erforderlich oder zumindest nützlich sein, wenn es um Maßnahmen geht, die flächenbezogene Nutzungsbeschränkungen mit sich bringen, um Umwelt- und Naturschutzerfordernissen zu genügen. Solche Pläne und Planprodukte finden sich beispielsweise im BNatSchG (Landschaftsplanung; Schutz besonderer Gebiete) und auch im WHG (Bewirtschaftungsplanung; Ausweisung von Wasserschutzgebieten).

Eine spezifische rechtlich abgesicherte Entwicklungsplanung für die Sicherung und Stärkung der Biodiversität in der Agrarlandschaft, die Regelungswirkungen für die landwirtschaftliche Bodennutzung beinhaltet und auch eine Sanierungsplanung für besonders belastete Gebiete, die infolge einer verursachergerechten Zuordnung Restriktionen für die landwirtschaftliche Bodennutzung beinhalten, gibt es demgegenüber gegenwärtig nicht, obwohl insbesondere auch das Unionsrecht entsprechende Gebietsausweisungen nahelegt bzw. verlangt. ${ }^{55}$ Dies gilt auch für die Integration geeigneter Gebiete zur besonders hervorgehobenen Entwicklung der Biodiversität in der Kulturlandschaft (,Entwicklungsgebiete“), wie sie von der Idee her in $\$ 25 \mathrm{Abs} .1 \mathrm{Nr} .3$ und $4 \mathrm{BNatSchG}$ für Biosphärenreservate schon formuliert ist.

Gemäß unserer Konzeption des Landwirtschaftsrechts bedarf es einer solchen Planung sowohl im Interesse von Sanierungs- als auch im Interesse von Entwicklungserfordernissen (z.B. im Rahmen der Biotopverbundplanung, o 20,21 BNatSchG, Art. 10 FFH-RL). In diesen Räumen kann eine - über die Grundpflichten hinausgehende - besondere Rücksichtnahme auf Umwelt- und Naturerfordernisse geboten sein und Ausgleichs- bzw. Förderleistungen rechtfertigen. ${ }^{56}$

\subsection{Ausweisung landwirtschaftlicher Belastungsgebiete}

Unter landwirtschaftlichen Belastungsgebieten verstehen wir solche, in denen die Leistungs- und Funktionsfähigkeit des Naturhaushaltes infolge der landwirtschaftlichen Bodennutzung schwer gestört und Sanierungsmaßnahmen deshalb notwendig sind. Von einer schweren Störung muss insbesondere dann ausgegangen werden, wenn im Wirtschaftsraum die grundwasserbezogenen Nitratgrenzwerte oder bodenbezogene Qualitätswerte überschritten sind. Auf der Landesebene sollten entsprechende Gebiete durch die dafür zuständige Behörde ausgewiesen und konkrete Maßnahmen zur Wiederherstellung der ökologischen Funktionsfähigkeit dieser Räume festgelegt werden.

46) VG München, Urt. v. 22.3.2019 - M 19 K 17.3738.

47) BVerfG, Beschl. v. 12.1.1967 - 1 BvR 169/63, BVerfGE 21, $73 / 82 \mathrm{f}$

48) Zur Ökologiepflichtigkeit (auch) des Waldeigentums Czybulka, NuR 2020, 73 m.w. N. und Czybulka., EurUP 1/2021, S. 2-21.

49) Siehe nur Wolff/Bachof/Stober, VerwR II, $\$ 56$ Rdnr. 6; Ehler, in: Erichsen/Ehlers (Hrsg.), VerwR, $\mathbb{\$} 1$ Rdnr. 71; Maurer, VerwR, $\$ 16$ Rdnr. 14; Köck, Pläne, in: GrVwR II, \$38, Rdnr. 10.

50) Der jüngste Agrarpolitische Bericht der Bundesregierung stammt aus dem Jahr 2019.

51) Köck, in: GrVwR II, $\$ 38, R d n r .44$

52) Siehe zur Einteilung der Pläne statt vieler: Köck, in: GrVwR II, $\$ 38, \mathrm{Rdnr} .55$.

53) Siehe nur die in Art. 4 Abs. 4 der Nitrat-Richtlinie genannten Aktionsprogramme.

54) Vgl. Möckel, in: Härtel (Hrsg.), Handbuch des Fachanwalts Agrarrecht, 2012, Kapitel 22, S. 820-846.

55) Vgl. Art. 10 FFH-RL und Art. 3 Abs. 2 Nitrat-Richtlinie.

56) Siehe unten zu 5 
Die Durchsetzung dieser Maßnahmen gegenüber den im betroffenen Raum wirtschaftenden Landwirten, obliegt der zuständigen Behörde. ${ }^{57}$ Gehen die notwendigen Maßnahmen über die allgemeinen Grundpflichten hinaus, sollte den betroffenen Landwirten ein Billigkeitsausgleich nach Maßgabe der Bereitstellung durch den Haushaltsgesetzgeber gewährt werden. Umgekehrt sollte aber auch berücksichtigt werden, wenn Sanierungsmaßnahmen in spezifischer Weise Vorteile für die im betroffenen Raum wirtschaftenden Landwirte begründen. Ob landwirtschaftliche Belastungsgebiete ausgewiesen werden, sollte nicht im Ermessen der Behörde stehen, sondern von allen verlangt werden können, die durch die Störung der ökologischen Funktionsfähigkeit in qualifizierter Weise betroffen sind. Dazu gehören beispielsweise auch die Wasserversorger, wenn infolge landwirtschaftlicher Tätigkeit die Trinkwasserversorgung aus dem Grundwasser erschwert wird. ${ }^{58}$

\subsection{Erhaltung und Stärkung der Biodiversität; Landschaftsentwicklung}

Die Erhaltung und Stärkung der Biodiversität sowie die Landschaftsentwicklung wird bereits durch ein Set von naturschutzrechtlichen Instrumenten, wie etwa die Landschaftsplanung oder den Schutz besonderer Gebiete, adressiert. Die Gebietskulissen besonderer Schutzgebiete und auch die Landschaftsplanung als ein vorbereitendes Instrument haben aber nicht ausgereicht, um die Biodiversitätsschutzziele zu erreichen. Insbesondere die Biotopverbundplanung ist auf naturschutzrechtlicher Grundlage defizitär geblieben.

Vor diesem Hintergrund erscheint es sinnvoll und notwendig, die Landschaftsplanung bzw. die auf den Landschaftsschutz und die Biodiversitätssicherung bezogenen Ziele und Grundsätze der Raumordnung durch ein zielverwirklichendes bundesweites Programm zu untersetzen, das für landschaftsstrukturelle und biodiversitätssichernde Maßnahmen der Landwirte Fördermittel bereitstellt. ${ }^{59}$ Die Programme müssen Umsetzungsfristen enthalten und $\mathrm{zu}-$ dem die Ergebnisse der Landschaftsplanung bzw. die Ziele und Grundsätze der Raumordnung umsetzen, weil nur diese Kopplung zusammen mit der Fristsetzung die Gewähr dafür bietet, in der Landschafts- und Biodiversitätsentwicklungspolitik substanziell voranzukommen. Maßnahmendurchführung und -pflege sind durch ein Monitoring auf ihre Effizienz zu überprüfen. Sollte das (freiwillige) Förderprogramm für die Verwirklichung der Planung nicht ausreichen, ist nach Maßgabe zwingender öffentlicher Interessen für die Durchsetzung des Programms Sorge zu tragen. Diese könnte beispielsweise durch die Aufnahme entsprechender Anordnungsbefugnisse ${ }^{60}$ und flankierender

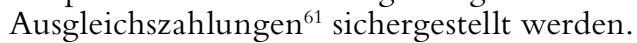

\section{Definition der landwirtschaftlichen Tätigkeit und landwirtschaftlichen Fläche}

\subsection{Landwirtschaftliche Tätigkeit}

Nach unserer Konzeption ist die Landschaftspflege ein wichtiger Aufgabenbereich der Landwirtschaft, auch wenn sie nicht mit der Gewinnung von Erzeugnissen verbunden ist. Dagegen sind die bisherigen Direktzahlungen ${ }^{62}$ (Erste Säule) an „landwirtschaftliche Tätigkeiten“ im konventionellen Sinne gebunden. Nach dem 10. Erwägungsgrund der VO 1307/2013 sollte es den Mitgliedstaaten freistehen, von Direktzahlungen an andere natürliche oder juristische Personen, deren landwirtschaftliche Tätigkeit marginal ist, abzusehen. [...]. Die Mitgliedstaaten sollten ferner von Direktzahlungen an natürliche oder juristische Personen absehen, deren landwirtschaftliche Flächen hauptsächlich auf natürliche Weise in einem für die Beweidung oder den Anbau geeigneten Zustand erhalten werden, die jedoch keine Mindesttätigkeit ausüben."
Diese Konzeption wurde durch das Greening mit seinen ökologischen Vorrangflächen teilweise aufgegeben. Für die neue GAP-Periode ist zu erwarten, dass das Greening abgeschafft wird und dann die Streitigkeiten bezüglich der Förderung „,naturbelassener Flächen“ wieder aufleben. Deshalb muss auch Landschaftspflege als „landwirtschaftliche Tätigkeit" anerkannt werden, wenn sie durch einen landwirtschaftlichen Betrieb oder Landschaftspflegeverband ausgeübt wird. Sollte eine Mindesttätigkeit zu bestimmen sein, ist sie so niedrig anzusetzen, dass die Vorgabe z. B. bei gelegentlicher Schafbeweidung, einmaliger Mahd oder Errichtung von Zäunen (z. B. als Wolfsschutz) eingehalten ist. Ein vergleichbares Problem besteht im Landpachtrecht, etwa für die Anlage und Pflege von Landschaftselementen. \$1 Abs. 2 LandpachtG versteht unter Nutzung die landwirtschaftliche Bodenbewirtschaftung zur Gewinnung pflanzlicher Erzeugnisse, zur Haltung oder Züchtung von Nutztieren oder zur Fischzucht.

Teile der Landwirtschaft lehnen das Konzept ab, wonach Naturschutz und Landschaftspflege nicht als Hemmnisse landwirtschaftlicher Tätigkeit verstanden werden sollten, sondern als ein der Produkterzeugung gleichwertiger Auftrag an die Betriebe. ${ }^{63}$ Dem liegt ein fatales Missverständnis zu Grunde: Die Tätigkeit der Landwirte beschränkt sich nicht auf die Produktion von Nahrungs- und Futtermitteln, die Haltung von Nutztieren oder die Erzeugung energetischer Biomasse, sondern sie wirkt sich unmittelbar auf Gemeinschaftsgüter, wie etwa die Biodiversität, den Wasserhaushalt und die Landschaft aus. ${ }^{64}$ Diese Umweltressourcen können nicht dem Ausschließlichkeitsanspruch des Eigentums unterworfen werden. Sie sind nicht individuell zuteilbar, sondern für alle da. Daraus folgt, dass sie nicht über den Markt zu steuern sind, sondern gemeinschaftliche Beschlüsse, also Rechtsregeln erfordern. ${ }^{65} \mathrm{Zu}$ physischen Umweltressourcen wie saubere Luft, sauberes Wasser, intakte Böden usw. tritt die belebte Umwelt hinzu, also Tier- und Pflanzenarten und ihre Lebensgemeinschaften (,Artenvielfalt“ bzw. „Biodiversität"). Diese Gemeinschaftsgüter besitzen zwei weitere Eigenschaften: Wie wir mit ihnen umgehen, betrifft auch künftige Generationen, und ihre Vernichtung ist irreversibel. Dass dies nicht sein darf, sondern die ,natürlichen Lebensgrundlagen“ zu schützen sind, bestimmt Art. 20a GG, wobei der Gesetzgeber die (in der Landwirtschaft ganz überwiegend privaten) Betreiber in die Pflicht nehmen muss, um das "Staatsziel“ $\mathrm{zu}$ erreichen. ${ }^{66}$ Geeignete Grundlage wäre das Landwirtschaftsgesetz, das eine entsprechend ,weite“ Definition der landwirtschaftlichen Tätigkeit beinhalten muss, v.a. um ein Förderungssystem $^{67} \mathrm{zu}$ legitimieren. Dieses hat ökonomisch und rechtlich einen anderen Ansatz als bestehende Kulturlandschaftsprogramme, die - oft mit Erfolg, aber nur dort, wo es sie gibt das Ziel der Biodiversität im ländlichen Raum verfolgen.

57) Siehe oben zu 2.4.3.

58) Dazu grundlegend: EuGH, Urt. v. 3.10.2019 - C-197/18, NuR 2019, 751 (Burgenländischer Wasserleitungsverband). Siehe dazu näher Köck/Henn, NVwZ 2020, 504-511.

59) Siehe unten zu 5

60) Siehe oben zu 2.4.3

61) Siehe unten zu 5.6

62) Nach der VO (EU) Nr. 1307/2013 vom 17.12.2013 mit Vorschriften über Direktzahlungen an Inhaber landwirtschaftlicher Betriebe im Rahmen von Stützungsregelungen der Gemeinsamen Agrarpolitik und zur Aufhebung der VO (EG) Nr. 637/2008 des Rates und der VO (EG) Nr. 73/2009.

63) Deutscher Verband für Landschaftspflege (DVL) o. Jg.: Gemeinwohlprämie - Umweltleistungen der Landwirtschaft einen Preis geben. Broschüre Ansbach, $11 \mathrm{~S}$.

64) Siehe oben zu 2.6.

65) Ostrom, 1990: Governing the commons. The evolution of institutions for collective action. Cambridge, U. K.

66) Näher Czybulka., EurUP 1/2021, S. 2-21.

67) Siehe unten zu 5 
Verräterisch ist, dass bisher Zahlungen als „Zuwendungen“, „Entschädigungen“, „Erschwernisausgleich“ u. ä. bezeichnet werden statt als Honorierung ökologischer Leistungen. Dahinter steckt die Vorstellung, dass einem Betrieb ein „Schaden“ entsteht, wenn er nicht intensiv unter Beeinträchtigung der Biodiversität wirtschaftet. Diesen „Schaden" gelte es auszugleichen, wobei darauf zu achten sei, dass der Landwirt daran nicht etwa verdiene - es dürfen nur Ertragsverluste und Mehraufwendungen erstattet werden. ${ }^{68}$ Ein Landwirt, der eine Blumenwiese ohne oder mit geringem Aufwand anbietet, verdiene keine Bezahlung; erhält er sie dennoch, so spricht man von einem ,Mitnahmeeffekt". Diese Konstruktion ist das Gegenteil dessen, was die Wirtschaftswissenschaft fordert: die Bewirtschaftung von Kollektivgütern nach Maßgabe ihres Wertes und die Setzung von Anreizen. ${ }^{69}$ Dass ein Landwirt an der Erzeugung von Weizen verdienen darf, ja soll, für die Erzeugung eines Kollektivgutes aber nur „,entschädigt“ " wird, zeigt (leider kaum ausrottbares) ökonomisches Unverständnis.

\subsection{Landwirtschaftliche Fläche}

Konsequenterweise muss die Definition ,landwirtschaftlicher Flächen“ im Landwirtschaftsgesetz diesem Verständnis von landwirtschaftlicher Tätigkeit angepasst werden. Denn sie ist entscheidend für das Fördersystem, soweit und solange dies wesentlich an die Fläche gekoppelt ist. Die Kommission schlägt folgendes vor: ${ }^{70}$ „Die entsprechenden Rahmendefinitionen von „Ackerland“, „Dauerkulturen“ und „Dauergrünland" sollten weit gefasst sein, damit die Mitgliedstaaten die Definitionen je nach örtlichen Bedingungen näher ausgestalten können. Die Rahmendefinition von „Ackerland" sollte so gefasst sein, dass die Mitgliedstaaten verschiedene Erzeugungsformen einschließlich der Agroforstwirtschaft sowie Ackerflächen mit Sträuchern und Bäumen darunter subsumieren können und die Aufnahme von Brachflächen vorgeschrieben ist, damit der entkoppelte Charakter der Interventionen gewährleistet ist. Die Rahmendefinition von „Dauergrünland“ sollte so gefasst sein, dass die Mitgliedstaaten weitere Kriterien festlegen und andere Arten als Gras oder andere Grünfutterpflanzen, die abgeweidet werden oder der Erzeugung von Futtermitteln dienen können, berücksichtigen können, unabhängig davon, ob sie tatsächlich zur Erzeugung genutzt werden oder nicht."

Dieser Vorschlag ist zum Grünland etwas kompliziert, geht aber in die hier vorgeschlagene Richtung. Weitere Klarstellungen sind erforderlich, z. B. dass wiedervernässte landwirtschaftliche Flächen, die beweidet werden oder mittels Paludikultur ${ }^{71}$ genutzt werden, Bestandteile der förderfähigen Hektarfläche sind..$^{72}$ Ähnliches gilt für verbindende Landschaftselemente i. S. v. Art. 10 FFH-RL und den Biotopverbund nach $\$ 21$ Abs. 5 BNatSchG.

\section{Leitlinien eines nationalen Fördersystems}

Das landwirtschaftliche Fördersystem gliedert sich in einen Bereich, für den nach wie vor die EU zuständig ist, und in einen zweiten Bereich, der in starkem Maße von nationalen Strategien abhängt. Dieser Bereich betrifft nicht nur die traditionelle zweite Säule der GAP, sondern erfasst auch Teile der ersten Säule. Die nachfolgenden Leitlinien beziehen sich ausschließlich auf diesen, den Mitgliedstaaten zugänglichen Bereich.

Eine Förderung der nachhaltigen Landwirtschaft zielt insbesondere auf ökologische Leistungen der Landwirtschaft mit Blick auf die Landschaftsentwicklung und den Schutz der Biodiversität. Die Förderung dieser Transformation hat ihren legitimen Platz jenseits der allgemeinen Grundpflichten. ${ }^{73}$ Von der Förderung zu unterscheiden sind Ausgleichsleistungen für Sonderlasten. Auch dieser Aspekt sollte im Landwirtschaftsgesetz geregelt werden; er betrifft besondere Einschränkungen der Landwirtschaft in Belastungs- und Entwicklungsgebieten. ${ }^{74}$

\subsection{Ausgangspunkt: Nationale Agrarförderung} in das Landwirtschaftsgesetz

Auf europäischer wie nationaler Ebene sind genügend Finanzmittel vorhanden, um die Leistungen der Landwirte für Umwelt und Biodiversität über die in den Betreiberpflichten geregelten ökologischen und betrieblichen Grundanforderungen hinaus dauerhaft zu vergüten. Es fragt sich, wo die Fördergrundsätze gesetzlich zu verorten sind. Maßgeblich ist bislang auf nationaler Ebene das Gesetz über die Gemeinschaftsaufgabe ,Verbesserung der Agrarstruktur und des Küstenschutzes" (GAKG). Es ist mit den EU-Milliarden aus dem ELER-Fonds verknüpft, die von den Mitgliedstaaten zu kofinanzieren sind. Seine gegenwärtigen Zielstellungen sind nur teilweise zur Übernahme in ein Landwirtschaftsgesetz geeignet: Gefördert werden u. a. „Maßnahmen einer marktund standortangepassten sowie umweltgerechten Landbewirtschaftung einschließlich Vertragsnaturschutz und Landschaftspflege“ sowie „Maßnahmen zur Förderung der Infrastruktur ländlicher Gebiete im Rahmen der Gemeinsamen Agrarpolitik der Europäischen Union“".75 Es spricht viel dafür, die ,,passenden“ Zielstellungen ${ }^{76}$ und Maßnahmen in das neue Landwirtschaftsgesetz zu übernehmen.

\subsection{Leitlinien der Förderung}

Vordringlich ist die Bewahrung der Reste hoher Agrobiodiversität und auf größerer Fläche die (Wieder-)Herstellung und Pflege landwirtschaftlicher Flächen mit (gegenüber jetzt) erhöhter Biodiversität. Die Flächen sind größtenteils bekannt, die erforderliche Landschaftsentwicklung ist nach den dargelegten planerischen Grundsätzen ${ }^{77}$ umzusetzen.

68) Angeblich auf Geheiß der WTO, in Wirklichkeit in vorauseilendem Gehorsam ihr gegenüber, um auf Drängen von Rechnungshöfen den Naturschutz möglichst billig zu machen. Die WTO schreitet nur ein, wenn sich ein Land beschwert, was nicht der Fall und nicht zu erwarten war (Derissen, Chancen eines marktwirtschaftlich orientierten Naturschutzes. Die Honorierung ökologischer Leistungen aus der Sicht der WTO, Diplomarbeit Greifswald, 2007; Geisbauer/Hampicke, Ökonomie schutzwürdiger Ackerflächen. Was kostet der Schutz von Ackerwildkräutern?, Greifswald, 2007, 2. Aufl. 2013, Broschüre). In der Praxis wird die Regel unterlaufen, um genügend Teilnehmer an Kulturlandschaftsprogrammen zu gewinnen. Gemäß Geisbauer \& Hampicke 2013) wird für Ackerrandstreifen etwa doppelt so viel bezahlt wie es die reinen Kosten erforderten.

69) Hampicke, Agricultural conservation measures - Suggestions for their improvement. German Journal of Agricultural Economics 62 (2013), 203-214.

70) Vorschlag der Kommission für eine Verordnung mit Vorschriften für die Unterstützung der von den Mitgliedstaaten im Rahmen der GAP zu erstellenden und durch den Europäischen Garantiefonds für die Landwirtschaft (EGFL) und den Europäischen Landwirtschaftsfonds für die Entwicklung des ländlichen Raums (ELER) zu finanzierenden Strategiepläne (GAP-Strategiepläne) und zur Aufhebung der VO (EU) Nr. 1305/2013 sowie der VO (EU) Nr. 1307/2013 \{SEC(2018) 305 final $\}-\{$ SWD(2018) 301 final $\}$, S. 13.

71) Wichtmann/Schröder/Joosten (Hrsg.), Paludikultur - Bewirtschaftung nasser Moore, 2016.

72) Die Sonder-Amtschefkonferenz am 4.7.2018 in Berlin, Stand 20.7.2018, Protokoll S. 4. hat den Bund gebeten, sich in diesem Sinne auf europäischer Ebene für eine klarstellende Begriffsdefinition ,landwirtschaftlicher Fläche“ und „Dauergrünland“ einzusetzen.

73) Siehe oben zu 2.2.

74) Siehe oben zu 3.2.

75) 51 Abs. 1 Nr. 2 und Nr. 7 GAK-Gesetz.

76) Vgl. den ,,allgemeinen Grundsatz“ des $₫ 2$ Abs. 1 Nr. 2 GAKG, wonach die Erfüllung der Gemeinschaftsaufgabe dazu dient, „die nachhaltige Leistungsfähigkeit ländlicher Gebiete, deren integraler Bestandteil eine umwelt- und ressourcenschonende Land- und Forstwirtschaft ist, zu gewährleisten."

77) Siehe oben zu 3.3. 
Gewiss wären besonders von großen Betrieben Beiträge auf eigene Kosten $\mathrm{zu}$ verlangen, eine durchgreifende Lösung des Problems auf diese Weise ist jedoch unrealistisch. Die Wiederherstellung der spezifisch agrarlandschaftlichen Artenvielfalt und schon die Bewahrung ihrer Reste verlangen extensive und traditionelle Formen der Landnutzung, die heute so unwirtschaftlich sind, dass sie jeden Betrieb, der sich ihnen auf eigene Kosten mehr als nur marginal widmete, schnell in den Ruin treiben würden. Wie später gezeigt, ist das „Abkaufen“ verteilungspolitisch unproblematisch. Die Summen, um die es geht, sind hoch für den landwirtschaftlichen Betrieb, aber trivial in gesamtwirtschaftlichen GröBen. Für den Landwirt hat die Förderung von BiodiversitätsMaßnahmen den Vorteil, dass sie für ihn kalkulierbar und verlässlich ist, unabhängig von Weltmarktpreisen. Leitlinie des hier vorgestellten Fördersystems ist die angestrebte Dauerhaftigkeit aller Biodiversitäts-Maßnahmen.

Daneben können auch umwelt- und tierschutzbezogene Verbesserungen der bisherigen Praxis durch Unterstützung betrieblicher Investitionen in Form von Zuschüssen oder Darlehen herbeigeführt werden, die ,,an sich“ einzuhaltende Betreiberpflichten darstellen, aber von den Landwirten allein nicht finanziert werden können (Anwendung des Gemeinlastprinzips für eine Ubergangszeit). Daneben kann man über Förderungen nachdenken, die der zunehmenden Entfremdung von Landwirtschaft und Gesellschaft entgegenwirken, insbesondere bei Ausbildung, wissenschaftlicher Forschung, Infrastruktur und Kontaktpflege im regionalen Bereich.

Um die Förderungen auf EU- und nationaler Ebene widerspruchsfrei zu handhaben, bedarf es Festlegungen der Begriffe „landwirtschaftliche Tätigkeit" und „landwirtschaftliche Fläche“. ${ }^{78}$

\subsection{Akzeptanz und Wirksamkeit}

Für Akzeptanz und Wirksamkeit des Fördersystems ist entscheidend, dass jeder Landwirt an den Förderprogrammen ohne erheblichen bürokratischen Aufwand teilnehmen kann. Es ist ein oft vernommener Vorwurf, dass insbesondere europäische Förderungen und der Vertragsnaturschutz zu bürokratisch seien und sich der Aufwand deshalb nicht lohne. Insbesondere werden auch „Kleinlichkeiten“ z.B. bei der Vermessung und Anrechnung von ökologischen Vorrangflächen moniert. Ganz im Gegensatz zu jahrzehntelanger nationaler Laschheit bei der Regelung von Düngung und Pestizideinsatz mit äußerst negativen Folgen wird dort ,genau hingesehen“. Dabei wurde gezeigt, dass Konflikte selten auf vertragswidrigem Verhalten der Landwirte und viel mehr auf Unklarheiten der ELER-Vorschriften und auf eine Überforderung lokaler Behörden zurückzuführen sind. ${ }^{79}$

Antragsverfahren können vereinfacht (siehe die ,Standardmaßnahmen"), aber es kann nicht auf Erfolgskontrollen verzichtet werden. Dabei sollte das europäischen Inventarund Kontrollsystem eingesetzt werden. Es deckt weit mehr Missbrauchsfälle auf als nationale Behörden. ${ }^{80}$ Durch die digital erfassten Flächen und Daten lassen sich Zahlungen und Kontrollen stark vereinfachen. Dies schlägt auch die Kommission vor: „Die Mitgliedstaaten werden die meisten Förderbedingungen auf nationaler Ebene festlegen und somit auf ihre besonderen Gegebenheiten zuschneiden können. Gleichzeitig soll der mit Kontrollen verbundene Verwaltungsaufwand reduziert werden, indem die direkte Verbindung zwischen den Förderbedingungen auf EU-Ebene und den Endbegünstigten begrenzt wird." 81

\subsection{Verwaltungszuständigkeiten für die Förderung}

Bei der Kompetenzverteilung für die Förderung sollten die Landwirtschaftsbehörden für betriebliche Förderungen im Tierschutz- und Umweltbereich zuständig sein (z. B. Stallumbau wegen Tierschutzanforderungen, Investitionszuschüsse für Filter oder Ausbringungstechnik) sowie für Biodiversitätsmaßnahmen außerhalb besonders geschützter
Gebiete (Standard-Förderprogramme, Landschaftspflege, Landschaftselemente). Die Naturschutzbehörden wären zuständig für spezifische Artenschutzprogramme (außerhalb der "Standardmaßnahmen") und Biodiversitäts-Maßnahmen innerhalb von Naturschutzgebieten.

Das flächenmäßig größte Problem stellen die Förderung und Kontrolle der landwirtschaftlichen Bodennutzung in Natura 2000 Gebieten dar. Hier werden die Vorgaben seit Jahrzehnten massiv missachtet. ${ }^{82}$ Durch eine wesentlich höhere Förderung extensiver Grünlandnutzung und die Bezahlung für die Pflege von Halbkulturlandschaften ist zwar eine Verbesserung der Situation zu erwarten, aber die Kontrollaufgabe übersteigt bei weitem die Kapazitäten der Naturschutzbehörden. Es bietet sich eine Kooperation zwischen Landwirtschafts- und Naturschutzbehörden an. Geeignete Institutionen wie Biologische Stationen, Landschaftspflegeverbände oder Stiftungen sind zu beteiligen.

Förderfähige „Standardmaßnahmen“ für die Bereiche Acker, Grünland und Landschaftselemente wurden seit langem entwickelt ${ }^{83}$ und sind nachfolgend mit ihren Kosten (beispielhaft) näher ausgeführt. Uber die Durchführung der Standardmaßnahmen hinaus ist keine Flächennutzung erforderlich. Die Bezahlung der Leistungen erfolgt an Kosten orientiert mit einer pragmatischen Anreizkomponente. ${ }^{84}$

Es ist zu überlegen, ob auch für Sonderkulturen förderfähige Standardmaßnahmen eingeführt werden können und ob die Pflege von Halbkulturlandschaften (z.B. Kalkmagerrasen, Heiden) und ähnlich anspruchsvollen Strukturen (Beweidung, maschinelle Pflege) in der Vergütung standardisiert werden kann, wenn sie im eigenen Betrieb stattfindet. ${ }^{85}$ Genaue Vollkostenkalkulationen z.B. für die Schafhaltung auf Kalkmagerrasen haben eine Mindestförderung vom mehr als $700 €$ pro ha und Jahr ergeben. ${ }^{86}$

\subsection{Kosten der Förderung von Biodiversität, Standardmaßnahmen}

Während die Kosten spezifischer Maßnahmen im Allgemeinen unterschätzt werden, werden die Kosten eines Program-

78) Siehe oben zu 4.

79) Kannegießer/Trepmann, Neustart für den ELER, in: Deutsche Vernetzungsstelle Ländliche Räume (Hrsg.), LandInForm 4.16, 2016, S. $44 \mathrm{f}$

80) Martinez, DVBl. 2020, 1186, 1191 sowie EuGH, Urt. v. 3. 10.2019, C-197/18, NuR 2019, 751 - Burgenländischer Wasserleitungsverband und Köck/Henn, NVwZ 2020, 504-511.

81) Vorschlag der Kommission für eine Verordnung mit Vorschriften für die Unterstützung der von den Mitgliedstaaten im Rahmen der GAP zu erstellenden und durch den Europäischen Garantiefonds für die Landwirtschaft (EGFL) und den Europäischen Landwirtschaftsfonds für die Entwicklung des ländlichen Raums (ELER) zu finanzierenden Strategiepläne (GAP-Strategiepläne) und zur Aufhebung der VO (EU) Nr. 1305/2013 sowie der VO (EU) Nr. 1307/2013 \{SEC(2018) 305 final $\}-\{$ SWD(2018) 301 final\}, S. 13.

82) Vgl. die mit Gründen versehene Stellungnahme der Kommission vom 12. 2. $2020 \mathrm{im}$ Vertragsverletzungsverfahren Nr. 2014/2262, betreffend die Bundesrepublik Deutschland.

83) Etwa Becker/Muchow/Schmelzer, in: Stiftung Rheinische Kulturlandschaft (Hrsg.), AgrarNatur-Ratgeber, 3. Aufl. 2020, 220 S.

84) Die dargestellte Kostenrechnung und eine an sie gelehnte Politik sind natürlich noch lange keine rigorose Umsetzung der Theorie der Kollektivgüter in die Praxis. Weder ist von aggregierter Zahlungsbereitschaft noch von Lindahl-Preisen noch von $\mathrm{Pa}$ reto-Optimalität die Rede.

85) Größere Pflegemaßnahmen werden wohl zumeist auszuschreiben sein.

86) Berger, Leistungen und Kosten zur Hüteschafhaltung mit Stallablammung und Lämmermast im benachteiligten Gebiet. Manuskript, 9 S., 2011; Umfassend Hampicke, Fachgutachten über die Höhe von Ausgleichszahlungen für die naturnahe Bewirtschaftung landwirtschaftlicher Nutzflächen in Deutschland. Im Auftrag der Umweltstiftung Michael Otto, überarbeitete und aktualisierte Fassung. Broschüre Hamburg, 2014. 
Tabelle 1 Kosten eines deutschlandweiten Programms zur Förderung der Biodiversität ${ }^{87}$

\begin{tabular}{lccc}
\hline & Fläche, ha $€ /$ ha.a & Mio. $€ / \mathbf{a}$ \\
\hline $\begin{array}{l}\text { Halbkulturlandschaft und } \\
\text { Traditionsgrünland }\end{array}$ & 1000000 & 550 & 550 \\
Grünlandextensivierung & 400000 & 1200 & 480 \\
Ackerwildkrautschutz & 150000 & 400 & 60 \\
$\begin{array}{l}\text { Strukturelemente } \\
\text { in der Ackerlandschaft }\end{array}$ & 630000 & 800 & 500 \\
Zusammen, rechnerisch & 2180000 & & 1590 \\
Zusammen, mit Aufrundung & & & 2000 \\
\hline
\end{tabular}

mes mit Standardmaßnahmen zur Förderung der Biodiversität völlig überschätzt. Das in Tabelle 1 zusammengefasste deutschlandweite Initiativ-Programm enthält vier Teile:

1. Das noch artenreiche Grünland (etwa ein Fünftel des gesamten) plus die Biotope der Halbkulturlandschaft sind (überwiegend durch Beweidung) zu pflegen. Der Kostenansatz in Höhe von $550 € /$ ha ist ein Mittelwert aus der z. T. teureren Bewirtschaftung der Halbkulturlandschaften und der billigeren des Traditionsgrünlandes.

2. $10 \%$ des heute intensivierten Grünlandes sind durch Rücknahme der Düngung und weitere Grünlandextensivierung in traditionelle „Fettwiesen“ „,zurück“ zu entwickeln, um nach dem erfolgreichen Vorbild in der Eifel ${ }^{88}$ Futter für das Jungvieh von Milchkuhhaltungen zu liefern (Tabelle 1 Stichwort Grünlandextensivierung auf weiteren $400000 \mathrm{ha}$ ).

3. $10 \%$ des ertragsärmsten Quartils des Ackerlandes ist unter Verzicht auf hohe Erträge mit den Zielen lebensfähiger Bestände von Wildkräutern, am Boden brütender Vogelarten und weiterer Arten zu bewirtschaften. Diese ebenfalls als Standardmaßnahme zu konzipierende Biodiversitätsmaßnahme ${ }^{89}$ verursacht Kosten in Höhe von $400 € /$ ha.

4. In hoch produktiven Börden sind sieben Prozent der Fläche, das sind etwa 630000 ha, in Strukturelemente umzuwandeln.

Zur Erläuterung: Bei Dauergrünland führt Bio-Landbau mit $110-150 \mathrm{~kg}$ Stickstoff pro ha und Jahr (organisch gedüngt) nicht zum gewünschten Artenreichtum der Grasländer. Traditionelle Frischwiesen erhielten früher Stallmist von $50-60 \mathrm{~kg}$ pro Hektar (sog. Fettwiesen) und wiesen 30-50 Pflanzenarten auf 10-15 $\mathrm{m}^{2}$ auf. Noch extensiver genutzte Grasländer (überwiegend in Mittelgebirgen und Alpenvorland) weisen weit mehr schutzwürdige Grünlandarten auf und sind entsprechend wertvoller. In Deutschland sind noch ca. 900000 ha Grünland für Biodiversität und Artenschutz interessant. ${ }^{90}$ Wenn all diese Flächen als Standardmaßnahme „Extensive Wiesen“ bzw. „Extensive Weiden" ${ }^{91}$, übergangsweise ${ }^{92}$ zusätzlich ,als Einstieg“" mit $200 € /$ ha und Jahr gefördert würden, sobald sie 40 Pflanzenarten auf $10-15 \mathrm{~m}^{2}$ aufweisen, ergäbe dies für Deutschland die (unglaublich geringe) Summe von 180 Mio. $€$ jährlich. Jeder Landwirt könnte an Standardmaßnahmen auf einfache Anzeige hin teilnehmen, die Kontrolle wäre ganz simpel. Den Weg über Ausmagerung etc. könnte jeder Landwirt selbständig gestalten.

Tabelle 1 fasst Maßnahmen, Flächenumfänge und Kosten des Programms zusammen. Mit einer großzügigen Aufrundung zugunsten von Kostensteigerungen (besonders bei Ackerflächen) sowie zur Gewährung von Anreizen ergeben sich zwei Milliarden $€$ pro Jahr. In der Anlaufphase dürften deutlich weniger Mittel abgerufen werden. Die Maßnahmen könnten über die Erste Säule der GAP (als „Konditionalität" oder „Ecoscheme“), aber auch auf andere Weise oder national gefördert werden. Bei einer angedachten Un- tergrenze von Ecoschemes von 30\% für die Erste Säule, wäre bereits durch diese Umschichtung die Anschubfinanzierung gesichert. Die restliche Summe müsste keineswegs neu erhoben werden, sondern wäre weitgehend, wenn nicht sogar vollständig durch Umlenkung schon vorhandener, aber fragwürdig allokierter Mittel zu gewinnen. Fällt diese Summe im Vergleich mit den wichtigsten Agrarbudgets (Erste Säule fünf Milliarden, Zweite Säule zweieinhalb Milliarden) durchaus ins Gewicht, so beträgt die Summe weniger als ein Promille (nicht Prozent) des jährlichen Brutto-Inlandsproduktes.

\subsection{Ausgleichszahlungen}

Von den Förderleistungen $\mathrm{zu}$ unterscheiden sind Ausgleichszahlungen. Ausgleichszahlungen sollen besondere Belastungen ausgleichen, die Landwirte jenseits der allgemeinen Grundpflichten treffen. Gesetzliches Vorbild dafür sind die Ausgleichszahlungen für besondere Einschränkungen in Wasserschutzgebieten ( $\$ 52$ Abs. 5 WHG), die zwar als Verstoß gegen das Verursacherprinzip kritisiert worden sind, ${ }^{93}$ aber gerechtfertigt werden können, weil Zahlungen dieser Art nach der hier vertretenen Konzeption erst jenseits der allgemeinen Grundpflichten in Betracht kommen. Ausgleichszahlungen sind nicht gleichzusetzen mit der eigentumsverfassungsrechtlichen Kategorie der ausgleichspflichtigen Inhaltsbestimmung des Eigentums, sondern setzen im Vorfeld an (Billigkeitsausgleich; Härteausgleich).

\section{Schlussbetrachtung}

Dem Landwirtschaftsgesetz aus dem Jahre 1955 liegen bis heute die Vorstellung der Landwirtschaft und der Agrarstruktur der 1950er Jahre zugrunde. Entfaltete es zum Zeitpunkt des Erlasses noch eine Steuerungswirkung, ist diese nunmehr vollständig verblasst und das Landwirtschaftsgesetz zu einem Beispiel für Symbolgesetzgebung degradiert. Diese fehlende Steuerungswirkung ist nicht durch andere legislative Akte kompensiert worden. Unter dem Vorwand marktwirtschaftlicher Freiheit oder mangelnder Gesetzgebungskompetenz hat der Gesetzgeber bis heute die Festlegung der Gemeinwohlaufgaben der Landwirtschaft gescheut. Unter den Gemeinwohlanliegen ragt die ökologische Verantwortung der Landwirtschaft heraus. Sie fußt nicht nur auf der Staatszielbestimmung des Art. 20a GG, sondern bereits darauf, dass die Landwirtschaft selbst von biologischen Prozessen abhängig ist und daher auf ein ökologisch intaktes Umfeld zwingend angewiesen ist. Die ökologische Verantwortung ist damit ein integraler Bestandteil der Landwirtschaft und nicht ein fremdes Element, das ihr auferlegt wird. Landwirtschaft kann des Weiteren nur als nachhaltige Landwirtschaft definiert werden; eine nicht nachhaltige Bodenbewirtschaftung ist eine ökologische und betriebswirtschaftliche Selbstzerstörung. Die deutsche Landwirtschaft hat aber in den letzten Jahrzehnten gezeigt, dass sie mit der Aufgabe überfordert war, diese Nachhaltigkeit zu definieren und damit auch zu gewährleisten.

87) Hampicke, Fachgutachten über die Höhe von Ausgleichszahlungen für die naturnahe Bewirtschaftung landwirtschaftlicher Nutzflächen in Deutschland. Im Auftrag der Umweltstiftung Michael Otto, überarbeitete und aktualisierte Fassung, Broschüre Hamburg, 2014.

88) Schumacher, in: Czybulka/Köck (Hrsg.), Landwirtschaft und Naturschutzrecht, S. $47 \mathrm{ff}$.

89) Vgl. dazu Maßnahmen A 4a und A 4b AgrarNatur-Ratgeber, S. $132 \mathrm{ff}$.

90) Schumacher, in: Czybulka/Köck (Hrsg.), S. 52 f.

91) Siehe im Einzelnen AgrarNatur-Ratgeber, Maßnahmen G 1 und G 2, S. $152 \mathrm{ff}$

92) Solange die flächenbezogene Basisprämie gezahlt wird

93) Kloepfer, Umweltrecht, 4. Aufl. 2016, \$14, Rdnr. 289. 
Der Gesetzgeber ist daher angehalten, diese ökologische Verantwortung der Landwirtschaft aktiv durch ein kohärentes Agrarumweltrecht zu steuern, das einem einheitlichen Leitbild und einheitlichen Standards folgt. Das Instrument hierfür ist ein neues Landwirtschaftsgesetz als Bundesgesetz, wozu der Bund auch über die Gesetzgebungskompetenz verfügt. Zur Festlegung ist der parlamentarische Gesetzgeber zuletzt auch durch das neue Umsetzungsmodell der GAP verpflichtet, das wohl im Jahr 2023 in Kraft treten wird und von Deutschland eine einheitliche, bundesweite Festsetzung der ökologischen Eckpunkte der Landwirtschaft fordert. Schicken wir also das Landwirtschaftsgesetz (1955) nach 66 Jahren in den wohlverdienten Ruhestand und nutzen wir die Zeit bis $2023 \mathrm{zu}$ einer parlamentarischen und gesellschaftlichen Debatte über die ordnungsrechtlichen Vorgaben und die finanzwirksamen Rahmenbedingungen einer künftigen biodiversitätserhaltenden und nachhaltigen Landwirtschaft.
Open Access. Dieser Artikel wird unter der Creative Commons Namensnennung 4.0 International Lizenz veröffentlicht, welche die Nutzung, Vervielfältigung, Bearbeitung, Verbreitung und Wiedergabe in jeglichem Medium und Format erlaubt, sofern Sie den/die ursprünglichen Autor(en) und die Quelle ordnungsgemäß nennen, einen Link zur Creative Commons Lizenz beifügen und angeben, ob Änderungen vorgenommen wurden.

Die in diesem Artikel enthaltenen Bilder und sonstiges Drittmaterial unterliegen ebenfalls der genannten Creative Commons Lizenz, sofern sich aus der Abbildungslegende nichts anderes ergibt. Sofern das betreffende Material nicht unter der genannten Creative Commons Lizenz steht und die betreffende Handlung nicht nach gesetzlichen Vorschriften erlaubt ist, ist für die oben aufgeführten Weiterverwendungen des Materials die Einwilligung des jeweiligen Rechteinhabers einzuholen.

Weitere Details zur Lizenz entnehmen Sie bitte der Lizenzinformation auf http://creativecommons.org/licenses/by/4.0/deed.de.

Open Access funding enabled and organized by Projekt DEAL.

\title{
Umweltproduktrecht - Konturen, Systematik und praktische Bedeutung
}

\section{Gerhard Wiebe}

\begin{abstract}
(c) Springer-Verlag GmbH Deutschland, ein Teil von Springer Nature 2021.
\end{abstract}
Erst der sog. Abgasskandal scheint ein stärkeres Bewusstsein für das Umweltproduktrecht in jüngerer Vergangenheit wieder geweckt $z u$ haben. Dabei existiert diese höchst praxisrelevante juristische Teildisziplin schon seit längerem; die Rechtswissenschaft hat sie indes seit geraumer Zeit vernachlässigt. Dieser Beitrag lädt daher ein, sich mit den Grundkonturen und der Systematik des Umweltproduktrechts auseinanderzusetzen, um die dogmatische Erschließung voranzutreiben und den praktischen Zugriff darauf zu verbessern.

\section{Einleitung}

Der eingangs erwähnte Abgasskandal bildet nur ein Beispiel in einer Reihe von Fällen, die zu der Einsicht geführt haben, dass der Konsum von (Verbraucher-)Produkten sich auf die Umwelt und ihre Medien negativ auswirken kann. Diese beinahe banale Erkenntnis korrespondiert nicht nur mit medialen Berichten, wie etwa solchen über die weltweit stetig wachsenden Elektroschrottmassen ${ }^{1}$ oder über die steigende Plastikverschmutzung der Gewässer. ${ }^{2}$ Daneben manifestiert sie sich in der kontinuierlich zunehmenden Regulierung der Konstruktion, der Herstellung, des Vertriebs, der Nutzung und der Entsorgung von Produkten unter dem Gesichtspunkt des Umweltschutzes; ${ }^{3}$ ein allgemeines Umweltschutzgebot im Recht des technischen Produkts existiert freilich (noch) nicht. ${ }^{4}$ Diese (neue) Zweckrichtung hat in den letzten Jahren auffällig häufig Einzug in das Produktrecht gehalten: Während das klassische Produktsicherheitsrecht in erster Linie den Produktnutzer und dessen Sicherheit in den Blick nimmt, zielt das umweltbezogene Produktrecht vornehmlich auf den Schutz der Umweltmedien $\mathrm{ab}$, wenn beispielsweise das Inverkehrbringen

Dr. Gerhard Wiebe,

Rechtsanwalt bei der KPMG Law Rechtsanwaltsgesellschaft mbH, Berlin, Deutschland von Plastikstrohhalmen und anderen Einwegkunststoffprodukte verboten wird, um Gewässerschutz zu betreiben. ${ }^{5}$

Doch die Geschichte des Umweltproduktrechts gründet nicht erst auf der vergleichsweise jüngeren Regulierungswelle; den rechtspolitischen Gründungsstein formt vielmehr das am 7. Februar 2001 von der Europäischen Kommission vorgelegte Grünbuch zur Integrierten Produktpolitik (IPP). ${ }^{6}$ Das Ziel der IPP besteht darin, die in einem Lebenszyklus eines Produkts, quasi „,von der Wiege bis zur Bahre“7 (vom Design über die Rohstoffgewinnung bis zur Entsorgung) hervorgerufenen Umweltauswirkungen $\mathrm{zu}$ erfassen und $\mathrm{zu}$ minimieren. Zudem soll eine maßnahmenbedingte Verlagerung negativer Umweltauswirkungen von einer Lebensphase auf eine andere verhindert werden. Mitte 2003 stellte die EU-Kommission das Weißbuch „Integrierte Produktpolitik - Auf den ökologischen Lebenszyklus-Ansatz aufbauen“" vor, das Instrumente und Maßnahmen zur Umsetzung der IPP enthält. ${ }^{8}$

1) Erst kürzlich Forti/Baldé/Kuehr/Bel, The Global E-waste Monitor 2020: Quantities, flows, and the circular economy potential, 2020, Stand 18.3.2021, abrufbar unter https://collections.unu. edu/eserv/UNU:7737/GEM_2020_def_july1.pdf.

2) Siehe hierzu Komm., Mitt. v. 16.1.2018, Eine europäische Strategie für Kunststoffe in der Kreislaufwirtschaft, COM(2018) 28 final; näher dazu Falke, ZUR 2001, $314 \mathrm{ff}$.

3) Wegener, in: Wegener (Hrsg.), Enzyklopädie Europarecht Bd. 8: Europäische Querschnittpolitiken, 1. Aufl. 2014, \$3 Rdnr. 59.

4) Bauer, Das Recht des technischen Produkts, 2018, Rdnr. 381.

5) Richtlinie (EU) 2019/904 des Europäischen Parlaments und des Rates vom 5.6.2019 über die Verringerung der Auswirkungen bestimmter Kunststoffprodukte auf die Umwelt, ABl. 2019 L 155, S. 1

6) Kommission der Europäischen Gemeinschaften, Grünbuch zur integrierten Produktpolitik, KOM (2001) 68 endgültig.

7) Bauer, Das Recht des technischen Produkts, 2018, Rdnr. 379.

8) Komm., Mitt. v. 18. 6.2003, Integrierte Produktpolitik: Auf den ökologischen Lebenszyklus-Ansatz aufbauen, KOM (2003) 302 endg. 